\title{
Series Hybrid Electric Vehicle Simultaneous Energy Management and Driving Speed Optimization
}

\author{
Boli Chen, Simos A. Evangelou and Roberto Lot
}

\begin{abstract}
The energy management (EM) and driving speed co-optimization of a series hybrid electric vehicle (S-HEV) for minimizing fuel consumption is addressed in this article on the basis of a suitably modeled series powertrain architecture. The paper proposes a novel strategy that finds the optimal driving speed simultaneously with the energy source power split for the drive mission specified in terms of the road geometry and travel time. Such a combined optimization task is formulated as an optimal control problem that is solved by an indirect optimal control method, based on Pontryagin's minimum principle. The optimization scheme is tested under a rural drive mission by extensive comparisons with conventional methods that deal with either speed optimization only or EM strategies with given driving cycles. The comparative results show the superior performance of the proposed method and provide further insight into efficient driving.
\end{abstract}

\section{NOTATION}

Current

$l \quad$ Total Length of Drive Mission

$m$ Mass

$P \quad$ Power

$Q \quad$ Battery Capacity

$R \quad$ Resistance

SoC Battery State-of-Charge

SoH Battery State-of-Health

$s \quad$ Travelled Distance

$T$ Temperature

$v \quad$ Vehicle Velocity

$\eta \quad$ Efficiency

$\rho \quad$ Energy Recovery Factor

$\tau$ Torque

$\omega \quad$ Angular Speed

\section{Subscripts}

$b \quad$ Battery

$d c \quad \mathrm{DC} / \mathrm{DC}$ Converter

$e$ Engine

$g \quad$ Generator

$h$ Mechanical Brake

$\begin{array}{ll}i & \text { Inverter } \\ m & \text { Motor } \\ r & \text { Rectifier } \\ t & \text { Transmission } \\ v & \text { Vehicle }\end{array}$

\section{INTRODUCTION}

$\mathbf{E}$ LECTRIFICATION of personal transport is identified as an essential step towards addressing the climate change threat. Ongoing efforts relate to enabling reductions

B. Chen is with the Dept. of Electronic and Electrical Engineering at University College London, UK (boli. chenducl. ac.uk).

$\mathrm{S}$. A. Evangelou is with the Dept. of Electrical and Electronic Engineering at Imperial College London, UK (s.evangelou@imperial.ac.uk) .

R. Lot is with the Dept. of Engineering and the Environment at the University of Southampton, UK (Roberto. Lotesoton.ac.uk).

This research was supported by the EPSRC Grant EP/N022262/1. of energy consumed by cars by shifting from conventional fuel powertrains to more electrified ones. The end of the road is expected to be fully electric vehicles, however the penetration of such vehicles is hindered by the perceived sacrifice of easy and quick refueling that average consumers have enjoyed up to now with conventional vehicles. Hybrid electric vehicles (HEVs) offer a suitable alternative design that combines the range and refueling convenience of engines with the cleaner electric energy of electric propulsion systems. HEV powertrains usually contain two or more energy sources for propulsion, such as an internal combustion engine (ICE) and a battery, therefore they are more complex than their counterparts in conventional vehicles. There is a variety of architectures in which the sources and other components in the powertrain can be arranged to provide propulsion to the $\mathrm{HEV}$, such as series, parallel and series-parallel architectures, with further subcategories in each case. In this article, the focus is on series architecture HEVs (S-HEVs) which include extended-range electric vehicles, such as the BMW i3 range extender, the Nissan Note e-power and VIA Motors products.

In terms of powertrain operation, energy management (EM) control strategies are used to decide intelligently on how to provide energy to the total vehicle load from the multiple energy sources in HEV powertrains. Despite the commercial success of HEVs, better fuel economy for these vehicles is being sought. This can be suitably achieved by new EM control, which remains one of the main challenges for a HEV.

A large number of EM strategies, from rule-based to optimization-based, have been proposed in the literature [1][5], among which the optimization-based approaches are usually heavy in computations, even though they have relied on predefined speed profiles upon which the optimization is performed. Instantaneous optimization-based techniques, such as the equivalent consumption minimization strategy (ECMS), represent valid alternatives that reduce the amount of computational burden at the price of less accuracy [6], while heuristic strategies that rely on reproducing the power split features of optimization-based strategies [7], [8], usually offer a good compromise between real-time implementability and optimality. However, there is no optimality guarantee for the rule based methods, which mostly originate from intuition. Machine learning techniques have also been recently adopted for HEV energy management [9], [10]. However, the performance is highly dependent upon the DP solutions because the optimal EM method is learned from DP solutions via neural networks.

The present paper contributes a new EM strategy based on the combined and simultaneous optimization of two important 


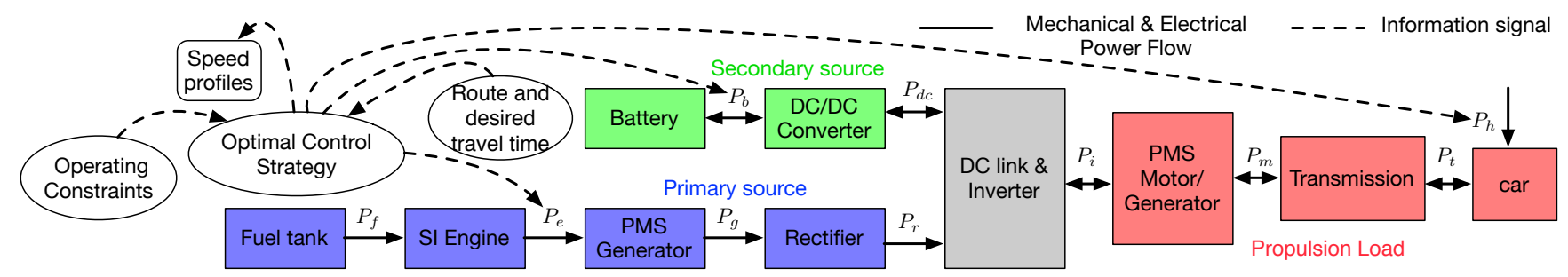

Fig. 1. Block diagram of the series HEV powertrain.

aspects of a hybrid vehicle: the longitudinal speed profile and the hybrid powertrain energy flow. The individual optimization of the latter of these two aspects is indeed well studied in the literature, as has been described in the previous paragraph. The individual optimization of the vehicle speed is also widely addressed for the purpose of minimizing fuel consumption in vehicles with conventional powertrains [11]. The combined optimization of the two aspects is a non-trivial step that produces a new EM methodology [12], which to the best of the authors' knowledge has not been addressed sufficiently. The proposed methodology can lead to the most efficient driving since both the vehicle speed and energy management are taken into account simultaneously in the optimization, in comparison to the methods that optimize these aspects separately and combine them a posteriori (this investigation is provided in this paper). The concerned problem is set up as an optimal control problem (OCP) in which the global minimum fuel consumption is sought by optimizing the speed profile and energy management power split throughout a given vehicle mission. An indirect optimal control tool is used in this paper to find the global optimum solution.

Despite the practical implications of following a given speed profile, which can be enabled by advanced cruise control systems, the proposed methodology overall offers a number of advantages in comparison to existing EM strategies that take speed profiles as known inputs: a) it removes the necessity of knowing a priori the driving cycle, which is otherwise usually unknown in practice, b) it also removes the necessity of predicting the future driving speed, which can otherwise lead to inaccurate speed profile predictions and consequently suboptimal powertrain energy management, c) it can lead to the most efficient driving as already mentioned, d) the drive mission is specified in terms of parameters than can be easily defined by the user or measured by a navigation system, such as the current location, the desired destination, and the path and time to destination, and e) the proposed scheme can be naturally extended to incorporate drive mission uncertainty, such as road traffic, traffic lights and so on, by reformulating it into a receding horizon problem, although this aspect is not studied in the present work.

The work in the paper extends in several aspects preliminary work on the simultaneous optimization of a series HEV speed profile and energy management in [13] and makes further important contributions: a) while the previous work considers solely the electrical behavior of the battery, in this work battery health and thermal effects are additionally taken into account and modeled, thus making it possible to reveal the impact of fuel efficiency optimization on the battery durability and safety, b) in contrast to a path with a constant legal speed limit and flat surface used in the previous work, a more realistic driving environment is created in this paper by developing a framework that takes into consideration the variable legal speed limit and road slopes of any given path, c) real driving speed data is collected in this work by a recently developed data acquisition device [14] and compared against optimized speed profiles for the same path to understand the nature and assess the importance of the speed optimization, d) the benefit of the newly developed combined and simultaneous optimization is demonstrated by comparisons with a twostep methodology that involves first conventional speed profile optimization followed by conventional powertrain power sharing optimization, and e) the conventional speed profile optimization is developed further to adopt it to HEV scenarios in which there is powertrain energy recovery, which leads to additional comparisons to those mentioned in part d).

The remainder of the paper is structured as follows. Section II introduces the vehicle and powertrain model, including all the powertrain component models, used in this work. The OCP and associated operational constraints are formulated in Section III. Section IV solves the OCP for a defined vehicle mission and shows the advantages of the proposed optimization scheme by comparative results. Finally, conclusions are drawn in Section V.

\section{Series Hybrid Electric Vehicle Model}

The proposed series HEV model represents a medium-size passenger car, and its powertrain configuration is sketched in Fig. 1. The S-HEV has three independent sources of power $P_{b}$, $P_{g}$, and $P_{h}$ (corresponding respectively to the battery, generator, and mechanical brakes), which can be mixed to obtain the desired values of vehicle speed and acceleration. The hybrid electric vehicle also allows regenerative braking that conveys braking power (negative $P_{t}$ ) through the transmission up to the battery. Optimal energy management fulfils minimization of fuel consumption by means of an appropriate power split.

The modeling of each component, which aims to capture the associated essential physical characteristics and in particular power losses, is presented next.

\section{A. Propulsion Load}

The propulsion load includes all the components from the inverter-driven permanent magnet synchronous (PMS) motor to the wheels, that request the load that has to be satisfied by the two sources (primary and secondary). 


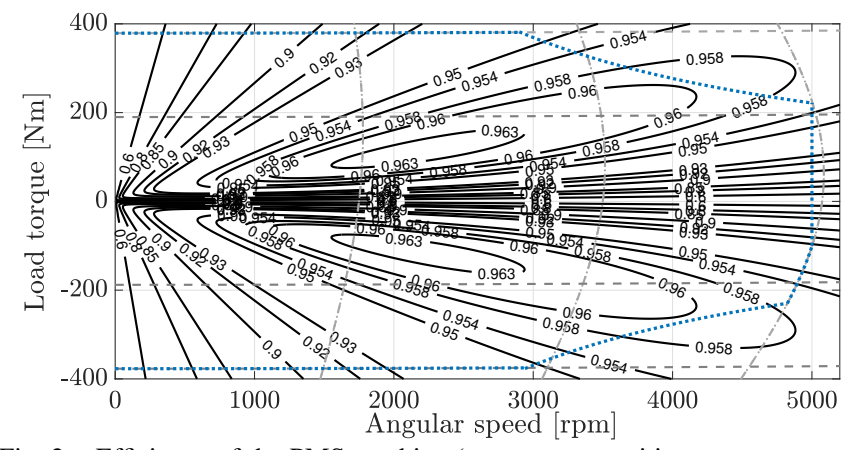

Fig. 2. Efficiency of the PMS machine (generator $=$ positive torque, motor $=$ negative torque) and operational bounds (blue dotted lines) for the reversible motor in terms of current, power, speed and voltage. The dashed horizontal lines correspond to constant currents (approximately equivalent to constant torques) and the vertical dash-dot curves correspond to constant voltages.

1) Three-phase DC to AC converter (Inverter): This work adopts a bidirectional pulse width modulated (PWM) inverter [15] in which the high frequency switching dynamics are neglected by averaging them out [16]. The efficiency of the energy transformation is therefore of interest, which is simply modeled as a constant efficiency factor $\eta_{i}$. Thus, the power balance of the DC link and inverter is described by $P_{i}=\eta_{i}^{\operatorname{sign}\left(P_{r}+P_{d c}\right)}\left(P_{r}+P_{d c}\right)$, where $\eta_{i}$ is adjusted according to the direction of the power flow.

2) PMS Motor/Generator: The behaviour of the PMS machine can be precisely described by a nonlinear dynamic model in $d-q$ reference frame [17]. This can be further simplified based on the assumptions that the dynamics of electromagnetic phenomena are much faster than mechanical ones and that the rotor inertia torque is reasonably smaller than the load torque, leading to a set of steady-state algebraic equations in terms of power flow [13]:

$$
\begin{aligned}
P_{m} & =\omega_{m} \tau_{l m}, \\
P_{i} & =\omega_{m}\left(\tau_{l m}+\tau_{d m}\right)-\frac{2}{3} R_{m} \frac{\left(\tau_{l m}+\tau_{d m}\right)^{2}}{\left(p_{m} \lambda_{m}\right)^{2}},
\end{aligned}
$$

where $P_{m}$ is the motor mechanical power and $P_{i}$ is the inverter electric power, $\tau_{l m}$ is the load torque and $\omega_{m}$ is the rotor angular speed. $\tau_{d m}$ is the total dissipation torque that collects a variety of electromagnetic and mechanical dissipation, including Eddy current, hysteresis, bearing and windage losses. In this work, $\tau_{d m}$ is assumed speed dependant, and approximated by a simple fitting technique to provide a realistic representation of the machine efficiency as compared to experimental data [16]. The stator resistance $R_{m}$, the rotor magnetic flux $\lambda_{m}$ and the number of pole pairs $p_{m}$ are constants specified in Table I given at the end of Section II-D. For the reversible PMS machine, it is convenient to adopt the convention that when it works as a generator, power (as well as the load torque) are positive, and the power is assumed negative when it works as a motor. In view of (1), the PMS motor/generator efficiency $\eta_{m}=\left(P_{m} / P_{i}\right)^{\operatorname{sign}\left(P_{i}\right)}$ may be explicitly evaluated as a function of the load torque $\tau_{l m}$ and velocity $\omega_{m}$, as shown in Fig. 2.

3) Transmission: A transmission with constant ratio $g_{t}=$ 10 is used and hence the relation between the motor angular

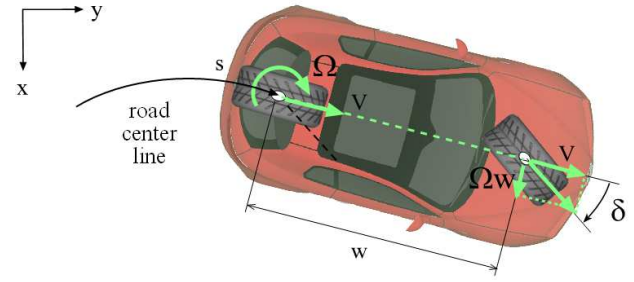

Fig. 3. Single-track, non holonomic, vehicle model.

speed $\omega_{m}$ and the vehicle forward speed $v$ is $\omega_{m}=\mathrm{g}_{t} v$. It is assumed that the transmission has a constant efficiency $\eta_{t}$. The bi-directional power flow is hence modeled with $P_{t}=\eta_{t}^{\operatorname{sign}\left(P_{m}\right)} P_{m}$, which adjusts the transmission efficiency according to the direction of power flow.

4) Brakes: Mechanical brakes are simply modeled as power withdrawal, that is as a source of negative power $P_{h}\left(P_{h} \leq 0\right.$ always). This power is dissipated as heat.

5) Vehicle longitudinal dynamics: The gross motion of the vehicle is described in terms of longitudinal speed $v$ and yaw rate $\Omega$, by using the single-track, non-holonomic vehicle model depicted in Figure 3. The longitudinal dynamics is described by the following differential equation:

$$
m_{v} \frac{d}{d t} v=F_{v}-F_{T}-F_{D}-F_{G},
$$

where $m_{v}$ is the overall vehicle mass, $F_{T}=f_{T} m_{v} g \cos \theta$, $F_{D}=f_{D} v^{2}$ and $F_{G}=m_{v} g \sin \theta$ are resistance forces due to rolling drag, aerodynamics drag and road grade $\theta$ respectively. $F_{v}$ is the longitudinal driving force determined by the transmission and brakes power

$$
F_{v}=\frac{P_{v}}{v}=\frac{P_{t}+P_{h}}{v} .
$$

The traveled distance $s$ is calculated by integrating the longitudinal speed:

$$
\frac{d}{d t} s=v .
$$

Assuming that the wheels are non-sliding, then the vehicle turning can be modeled as: $w \Omega=v \tan \delta$, where $\delta$ is the steering angle and $w$ the wheelbase. It is reasonable to assume that when driving on a single lane rural road, the driver remains approximately in the middle of its lane. The road is thus defined in terms of curvature of the road center $\Theta$ calculated from its Cartesian coordinates $(x, y)$ as a function of the travelled distance $s$ :

$$
\Theta(s)=\sqrt{\left(\frac{d^{2} x}{d s^{2}}\right)^{2}+\left(\frac{d^{2} y}{d s^{2}}\right)^{2}} .
$$

Therefore, the vehicle yaw rate $\Omega$ is simply the product of vehicle speed and road curvature as shown by $\Omega=v \Theta(s)$. This assumption is not representative of the driver behavior at intersections, hence sharp corners are conveniently converted into smoother profiles by properly filtering the curvature.

\section{B. Primary source}

1) Internal Combustion Engine: A common 1.8L spark ignition naturally aspirated engine is considered. The engine 
involves very complex dynamics but these are typically much faster than the powertrain energy flow dynamics of interest in the present work. Hence, a characterization of the average engine efficiency at steady-state operating conditions is adequate for the present purposes. The engine efficiency is derived from simulations with the industry standard engine and gas dynamics simulation software, Ricardo WAVE, which has been calibrated against experimental results. The efficiency $\eta_{e}\left(\omega_{e}, \tau_{e}\right)$ is expressed as a function of the crankshaft speed $\omega_{e}$ and brake torque $\tau_{e}$ at steady-state operating conditions, as shown in Fig. 4. The ICE is assumed to be "idle" for $\omega_{e} \leq$ $1000 \mathrm{rpm}$ and it has a maximum efficiency $\eta_{e, \max }=0.343$ for a brake torque of $151 \mathrm{Nm}$ at $2800 \mathrm{rpm}$.

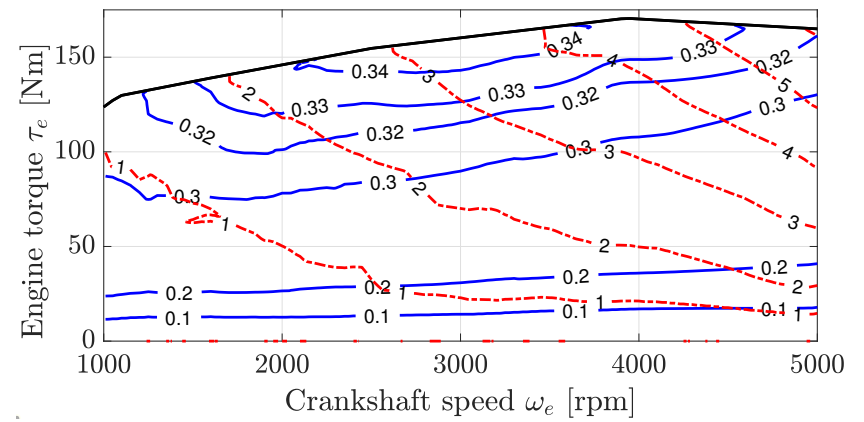

Fig. 4. Efficiency (blue solid) and fuel mass flow rate $[\mathrm{g} / \mathrm{s}]$ (red dashed) of the ICE with torque and speed operating points.

2) PMS Generator: The steady state equations (1) that describe the reversible PMS machine are applied to describe also the PMS generator, which works only in one direction. In the present work, both machines are assumed to have the same parameters. Therefore, the generator efficiency $\eta_{g}=P_{g} / P_{e}$ is a function of generator speed and load torque, immediately available from Fig. 2 .

3) Rectifier: The rectifier converts the generator $\mathrm{AC}$ into DC at constant voltage $V_{d c}$. A pulse width modulated (PWM) rectifier is adopted. Similarly to the inverter, it is simply modeled as a constant efficiency factor $\eta_{r}$, such that $P_{r}=\eta_{r} P_{g}$.

\section{Secondary source}

1) Battery: The Li-ion battery is modeled by a standard equivalent electrical circuit with purely ohmic impedance [4] representation, which captures the main battery dynamics by the differential equation of the battery state-of-charge $(\mathrm{SoC})$ :

$$
\frac{d}{d t} \mathrm{SoC}=-\frac{i_{b}}{Q_{\max }} .
$$

where $Q_{\max }$ is the battery capacity, and $i_{b}$ is the battery current, which is assumed positive during the discharge phase. Moreover, instead of having a nonlinear dependence on SoC, the battery open circuit voltage $V_{o c}$ is adequately approximated as a constant for the studied charge sustaining HEV. By considering the identity $P_{b}=V_{o c} i_{b}-R_{b} i_{b}^{2}$ that is inferred from the definitions of the battery closed circuit voltage $V_{b}=V_{o c}-R_{b} i_{b}$ and the battery output power $P_{b}=V_{b} i_{b}$, battery current $i_{b}$ can be solved as a function of $P_{b}$. Then, the solution is applied to reformulate (6) as

$$
\frac{d}{d t} \mathrm{SoC}=-\frac{V_{o c}-\sqrt{V_{o c}^{2}-4 P_{b} R_{b}}}{2 R_{b} Q_{\max }} .
$$

a) State-of-Health $(\mathrm{SoH})$ : Battery $\mathrm{SoH}$ is a measure that reflects the battery condition compared with a fresh battery. High fidelity SoH models, as the one utilized in a battery sizing problem in [18], are highly nonlinear due to the associated temperature effects and battery capacity drops because of SoH variations. However, in EM problems the optimization is solved over a horizon (distance of driving mission) that is a negligible fraction of the total distance to reach battery end-oflife, hence the SoH variation is very small. Consequently, there will be a negligible capacity drop and $Q_{\max }$ can be assumed to be constant. Additionally, as it will be shown, the battery temperature is maintained within a narrow band under active cooling conditions, and therefore temperature inlfuence can also be ignored. As such, a simple SoH model [19], capturing the essential features [20], is adequate and is utilized in this work:

$$
\frac{d}{d t} \mathrm{SoH}=\frac{-\left|i_{b}\right|}{2 N_{\text {cycle }} Q_{\max }},
$$

where $N_{\text {cycle }}$ is the number of charge-discharge cycles a new battery can take before it reaches $80 \%$ of the nominal energy capacity. By definition of the model, after this many chargedischarge cycles the $\mathrm{SoH}$ reaches zero.

b) Thermal model: For electric and hybrid vehicles, the prediction of the battery temperature is a vital process of the thermal management that is designed to optimize the driving performance. Accurate modeling of the thermal behavior requires high fidelity heat transfer models that are, however, inherently complex due to the increased dimension and the dependence on battery chemistry and composition [21]. In this paper, a simple model that captures the main thermal effects without significantly increasing the computation burden is adopted. The model is given by the following equation:

$$
\frac{d}{d t} T_{b}=\frac{R_{b} i_{b}^{2}-h_{c} A_{b}\left(T_{b}-T_{a m b}\right)}{c_{b} m_{b}}
$$

where $c_{b}$ and $m_{b}$ are respectively the mass thermal specific capacity and battery mass, $A_{b}$ is the heat transfer area of the battery, and $h_{c}$ is the heat transfer coefficient that is assumed constant for invariant cooling air mass flow rate. $T_{a m b}=25^{\circ} \mathrm{C}$ is the ambient temperature that is invariant in this study.

The characteristic parameters of the battery are chosen to emulate a realistic battery for a non plug-in HEV. The battery energy capacity is $1.5 \mathrm{kWh}$ (as in the Nissan note e-power). It corresponds to a small-sized battery and as such it must be capable of delivering continuous currents of $10 \mathrm{C}$ or more in practice for sufficient propulsion power [22]. Therefore, the maximum charging/discharging $\mathrm{C}$-rates are set to $10 \mathrm{C}$ and $20 \mathrm{C}$ respectively. However, repetitive charge-discharge cycles under such C-rate levels would rapidly make the battery wear out with deep discharges. Therefore, the battery SoC is limited in this work within the narrow range of $50 \%-80 \%$. The thermal parameters used are mainly obtained from [23], [24]. In particular, as mentioned in [23], the heat transfer coefficient $h_{c}$ may practically vary between $5 \mathrm{~W} / \mathrm{m}^{2} / \mathrm{K}$ (natural 
convection) and $65 \mathrm{~W} / \mathrm{m}^{2} / \mathrm{K}$ (forced-air convective cooling) depending on the cooling intensity. In this paper, $h_{c}$ is set to $25 \mathrm{~W} / \mathrm{m}^{2} / \mathrm{K}$, by which an environment with intermediate air cooling is assumed. The thermal specific capacity $c_{b}$ is set to $800 \mathrm{~J} / \mathrm{kg} / \mathrm{K}$, which is a validated value for a $\mathrm{Li}$-ion battery cell [23]. Furthermore, following the work in [24], it is assumed that the battery pack used in this work consists of 72 prismatic pouch cells (dimension: $16 \times 65 \times 151 \mathrm{~mm}$ ), formed in two arrays, with coolant passages (width of the coolant passages is $3 \mathrm{~mm}$ ) between each two cells to allow fresh air for circulation (see the configuration of the active air cooled battery system proposed in [24]). As such, the heat transfer area $A_{b}$ can be numerically determined. The remaining battery parameters are reported in Table I, provided in Section II-D.

2) $D C / D C$ converter: The battery voltage is amplified by a high-efficiency bidirectional DC/DC converter. Similarly to the rectifier the converter is modeled as a static element having constant efficiency $\eta_{d c}$ [25]. The bidirectional power conversion is thus described by $P_{d c}=\eta_{d c}^{\operatorname{sign}\left(P_{b}\right)} P_{b}$, in which $P_{b}$ is the battery power on the low voltage side, $P_{d c}$ is the battery power on the DC link side, and the efficiency is adjusted according to the direction of the power flow.

\section{Component integration}

In this Section a fuel consumption model is developed analytically by the efficiency analysis of the engine-generator system. Thereafter, all the individual components are assembled together to form the overall vehicle model, in which the accuracy of transient behavior and complexity are balanced.

1) Fuel consumption model: The mechanical integration of the ICE and the PMS generator enables the calculation of the efficiency $\eta_{f}$ of the transformation of fuel chemical power into electric power, which is simply the product of the engine and generator efficiencies: $\eta_{f}=\eta_{e} \eta_{g}$. The mechanical separation from the wheels allows a requested engine power to be supplied by freely choosing among different combinations of torque and speed, which optimize the engine-generator efficiency. As shown in Fig. 5, the relationship between the

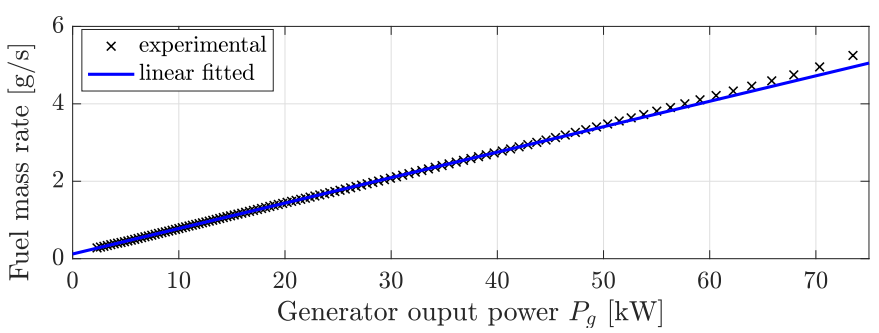

Fig. 5. Fuel mass rate with generator power, when the most efficient engine torque-speed operating point is followed at each power value.

engine fuel mass rate $\dot{m}_{f}$ and $P_{g}$ is approximately linear, provided the engine-generator unit is continuously operated along the locus of the most efficient torque-speed operating points. The fuel mass dynamic equation is therefore given by:

$$
\frac{d}{d t} m_{f}=\dot{m}_{f 0}+\frac{P_{g}}{q_{H V} \alpha_{f}}
$$

where $\dot{m}_{f 0}=0.12 \mathrm{~g} / \mathrm{s}$ is the fuel mass rate when the engine is idle, $q_{H V}=44 \mathrm{MJ} / \mathrm{kg}$ is the gasoline lower heating value and $\alpha_{f}=0.34$ is the equivalent factor of power transformation.

2) Overall powertrain model: In view of the power flow described already for each component, the vehicle power flow may be described as functions of the three independent power sources $P_{b}, P_{g}, P_{h}$, with the particular power output of the transmission given by:

$$
P_{t}=\left(\eta_{i} \eta_{m} \eta_{t}\right)^{\operatorname{sign}\left(\eta_{r} P_{g}+\eta_{d c}^{\operatorname{sign}\left(P_{b}\right)} P_{b}\right)}\left(\eta_{r} P_{g}+\eta_{d c}^{\operatorname{sign}\left(P_{b}\right)} P_{b}\right) .
$$

From a practical perspective, the three powers are not controlled directly in this work, but via the time-derivatives $j_{g}, j_{b}, j_{h}$ of the associated forces $F_{g}, F_{b}, F_{h}$, respectively, which have the dimensions of jerk, to ensure smooth controls and to avoid unrealistic jerky manoeuvres. Therefore, the three independent power sources are calculated by $P_{g}=F_{g} v, P_{b}=F_{b} v, P_{h}=F_{h} v$. Considering state variables $\mathbf{x} \triangleq\left[m_{f} \text {, SoC, } v, s, F_{g}, F_{b}, F_{h}\right]^{T}$ and control inputs $\mathbf{u} \triangleq\left[j_{g}, j_{b}, j_{h}\right]^{T}$, the following system is obtained by collecting (2), (4), (7), (10) and the dynamics of $F_{g}, F_{b}$ and $F_{h}$ :

$$
\frac{d}{d t}\left(\begin{array}{c}
m_{f} \\
\operatorname{SoC} \\
v \\
s \\
F_{g} \\
F_{b} \\
F_{h}
\end{array}\right)=\left(\begin{array}{c}
\dot{m}_{f 0}+P_{g} /\left(q_{H V} \alpha_{f}\right) \\
-\left(V_{o c}-\sqrt{V_{o c}^{2}-4 P_{b} R_{b}}\right) /\left(2 R_{b} Q_{\max }\right) \\
\left(P_{t}+P_{h}\right) /\left(m_{v} v\right)-\left(F_{T}+F_{D}+F_{G}\right) / m_{v} \\
v \\
m_{v} j_{g} \\
m_{v} j_{b} \\
m_{v} j_{h}
\end{array}\right) .
$$

Any other time-varying variables of the system, such as electric currents and voltages, torques and the vehicle power flow can be explicitly calculated by algebraic equations with respect to $\mathbf{x}$ and $\mathbf{u}$. In addition, battery state of health and temperature are calculated respectively from equations (8) and (9) for monitoring purposes. The main characteristic parameters of the vehicle model are summarized in Table I.

TABLE I

Main Vehicle Model Parameters

\begin{tabular}{lcl}
\hline \hline symbol & value & description \\
\hline$m_{v}$ & $1500 \mathrm{~kg}$ & vehicle mass \\
$f_{T}$ & 0.01 & tyre rolling resistance coefficient \\
$f_{D}$ & 0.47 & aerodynamics drag coefficient \\
$Q_{\max }$ & $5 \mathrm{Ah}$ & battery capacity \\
$R_{b}$ & $0.2056 \Omega$ & battery internal resistance \\
$V_{o c}$ & $300 \mathrm{~V}$ & battery open circuit voltage \\
$N_{c y c l e}$ & 2000 & battery life cycle \\
$m_{b}$ & $36 \mathrm{~kg}$ & battery mass \\
$c_{b}$ & $800 \mathrm{~J} / \mathrm{kg} / \mathrm{K}$ & battery mass specific heat capacity \\
$A_{b}$ & $1.56 \mathrm{~m}^{2}$ & battery heat transfer area \\
$h_{c}$ & $25 \mathrm{~W} / \mathrm{m}^{2} / \mathrm{K}$ & battery heat transfer coefficient \\
$\eta_{r}, \eta_{i}, \eta_{d c}$ & 0.96 & efficiency of converters \& inverters \\
$R_{m}$ & $90 \Omega$ & PMS machine stator resistance \\
$\lambda_{m}$ & $0.21 \mathrm{~Wb}$ & PMS machine rotor magnetic flux \\
$p_{m}$ & 6 & PMS machine number of poles \\
$\eta_{t}$ & 0.96 & efficiency of the transmission \\
$\mathrm{g}_{t}$ & 10 & transmission ratio \\
\hline \hline
\end{tabular}




\section{Optimal Control Problem Formulation}

Conventional energy management strategies are designed to find proper power component combinations that minimize fuel consumption for given vehicle speed profiles. However, the future driving speed is not known during real world driving. The idea proposed in this paper is based on a different concept, as shown in Fig. 1. The OCP is formulated by specifying the vehicle mission subjected to performance and operating constraints that must be satisfied. Instead of in terms of vehicle speed and acceleration, the drive mission is defined in terms of route and a specified traveling time. Therefore, according to the new methodology (OCP-Joint), the powertrain energy flow and speed profile along the route are jointly provided by the solution of the optimization.

An alternative methodology proposed in this work to solve the complete optimization problem is a two-step approach (OCP-2-step) that successively combines two conventional methods: 1) driving speed optimization (OCP-S), and 2) energy management optimization (OCP-EM), as shown Fig. 6. The OCP-2-step is related to conventional methods in which,

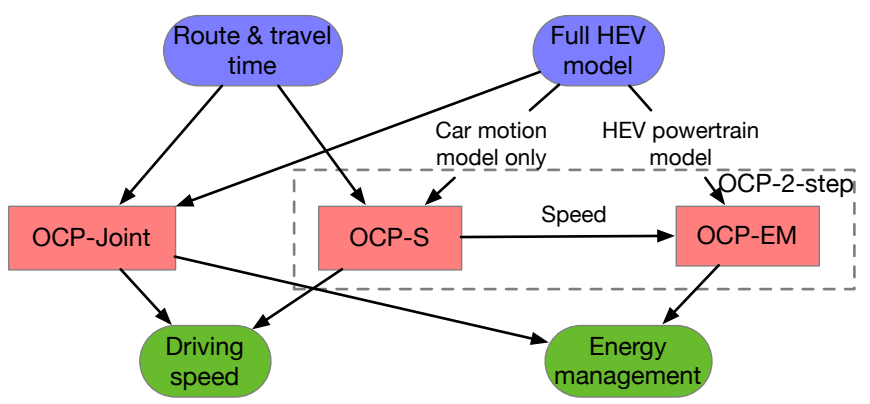

Fig. 6. Optimal control problems formulated for the series HEV.

for example, the power split is optimized for a given speed profile (that may or may not be an optimal speed profile), or the speed profile alone is optimized for a conventional (nonhybrid) vehicle. The present work compares the mentioned optimization approaches and exposes the benefits in vehicle fuel efficiency of conducting the combined optimization OCPJoint, which takes into account both the characteristics of the drive mission as well as the powertrain, as demonstrated later in Section IV. The general formulation of the OCPs studied in this paper is as follows:

$$
\begin{array}{ll}
\min _{\mathbf{u}} & J(\mathbf{x}, \mathbf{u})=\int_{0}^{t_{f}} \mathcal{L}(\mathbf{x}, \mathbf{u}, t) d t+\phi\left(\mathbf{x}\left(t_{f}\right)\right) \\
\text { subject to: } & \frac{d}{d t} \mathbf{x}=\mathbf{f}(\mathbf{x}, \mathbf{u}, t) \\
& \psi(\mathbf{x}, \mathbf{u}, t) \leq \mathbf{0} \\
& \mathbf{b}\left(\mathbf{x}(0), \mathbf{x}\left(t_{f}\right)\right)=\mathbf{0},
\end{array}
$$

where $J(\mathbf{x}, \mathbf{u})$ is the main objective. The vector $\mathbf{x}$ represents the system state vector, which evolves according to the differential equation $\dot{\mathbf{x}}=f(\mathbf{x}, \mathbf{u}, t)$, and the vector $\mathbf{u}$ represents the control input. The inequality constraints and the boundary conditions are taken into account by (12c) and (12d). Finally, $t_{f}$ denotes the total traveling time. In each OCP described below, the respective $J, \mathbf{x}, \mathbf{u}$, constraints and boundary conditions are different, and are specified. a) Vehicle speed optimization $(O C P-S)$ : Driving speed optimization in terms of fuel efficiency has been well studied in the literature for conventional vehicles [11]. In this section, this conventional approach is developed further and extended to HEVs by considering energy recovery during braking. A fixed energy recovery factor is defined as the ratio between regenerative braking power and the total braking power:

$$
\rho=P_{t} / P_{v}, \quad \forall P_{v}<0
$$

with $\rho \in[0,1]$. The boundary case $\rho=0$ corresponds to a conventional powertrain, since in the present work the fuel mass rate is linearly dependent upon the generator output power (see Fig. 5), while $\rho=1$ is associated with recovering all braking energy as in a highly hybridized or fully electric vehicle. The total driving/braking power is incorporated into the dynamic model of the longitudinal dynamics of the vehicle as the total driving/braking force $F_{v}$ (see (3)), as follows:

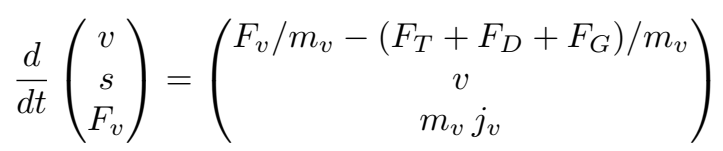

with $\mathbf{x} \triangleq\left[v, s, F_{v}\right]^{T}$. The OCP is formulated to find jerk input $\mathbf{u}=j_{v}$, associated with the total driving/braking power and consequently the speed $v$, which minimizes the net energy from the powertrain:

$$
J=\int_{0}^{t_{f}} P_{t} d t=\int_{0}^{t_{f}} \max \left(\rho F_{v}, F_{v}\right) v d t .
$$

The inequality constraints of this OCP are as follows. Inequality constraints are imposed on the speed and acceleration for driving safety and comfort. Specifically,

$$
0 \leq v \leq v_{\max }
$$

where $v_{\max }$ is the legal speed limit ${ }^{1}$. Instead of the commonly used ellipse of adherence of tires, the longitudinal and lateral acceleration are constrained within an acceleration diamond, as everyday drivers use accelerations remarkably smaller than adherence limits [26]. The constraint is described as follows:

$$
\left|\frac{F_{v} / m_{v}}{a_{x, \max }}\right|+\left|\frac{v \Omega}{a_{y, \max }}\right| \leq 1 .
$$

where $F_{v} / m_{v}$ and $v \Omega$ are respectively the longitudinal and the lateral acceleration applied by the driver, while $a_{x, \max }$ and $a_{y, \max }$ are their maximum allowed values. The total input power is limited according to

$$
v F_{v} \leq P_{v, \max },
$$

where $P_{v, \max }$ is determined according to the sum of the individual power limits of the engine and the battery branches. Finally, $j_{v}$ is bounded within $\pm 1 \mathrm{~m} / \mathrm{s}^{3}$.

The following boundary conditions are used to define the initial and terminal states:

$$
\begin{array}{cc}
s(0)=0, & s\left(t_{f}\right)=l, \\
v(0)=v\left(t_{f}\right)=v_{\min }, & F_{v}(0)=F_{v}\left(t_{f}\right)=0,
\end{array}
$$

\footnotetext{
${ }^{1}$ To avoid the issue of singularity when the power is divided by $v$, a nonzero lower bound is imposed, such that $v \geq v_{\min }$ with $v_{\min }$ a small constant.
} 
where $l$ is the length of the path and $t_{f}$ is expressed in terms of $l$ and an average speed for the mission.

b) Powertrain power-split optimization (OCP-EM): The general problem of optimizing the EM of an HEV while it follows a predefined speed, for example according to a given drive cycle, is standard, while it has also been specifically formulated in numerous prior studies as an OCP; see for example [3], [27]. Here such an EM OCP formulation is adapted and described for the proposed vehicle model.

For the given value of the vehicle speed and acceleration, the necessary driving force $F_{v}$ is uniquely determined by (2) whereas the power split is freely assigned. Based on the developed series HEV model, the dynamic model used for EM is part of (11), comprising only dynamics of $\mathbf{x}=\left[m_{f}, \mathrm{SoC}, F_{g}, F_{b}, F_{h}\right]^{T}$ (and $\mathrm{SoH}$ and $T_{b}$ for monitoring), subject to an algebraic constraint among $F_{b}, F_{g}$ and $F_{h}$ given by (3). The OCP is formulated to find $\mathbf{u}=\left[j_{b}, j_{g}, j_{h}\right]^{T}$ that minimizes $J=m_{f}\left(t_{f}\right)$, subject to multiple constraints that keep the operating conditions of the powertrain components inside their admissible range, as follows. The generator power is limited according to:

$$
0 \leq P_{g} \leq P_{g, \max }
$$

with $P_{g, \max }=86 \mathrm{~kW}$. The battery is constrained in terms of SoC and current:

$$
\mathrm{SoC}_{\min }<\mathrm{SoC}<\mathrm{SoC}_{\max }, \quad i_{b c}<i_{b}<i_{b d},
$$

where $\mathrm{SoC}_{\min }=0.5, \mathrm{SoC}_{\max }=0.8$, and $i_{b d}=100 \mathrm{~A}$ and $i_{b c}=-$ $50 \mathrm{~A}$ are determined by the discharging and charging $\mathrm{C}$ rate limits respectively. The PMS motor (and indirectly the inverter) is constrained by the operational limits as shown in Fig 2. Braking power is constrained to be negative:

$$
P_{h} \leq 0,
$$

and $j_{g}, j_{b}, j_{h}$ are bounded within $\pm 1 \mathrm{~m} / \mathrm{s}^{3}$.

The following boundary conditions are imposed to complete the formulation:

$$
\begin{aligned}
& \operatorname{SoC}(0)=\operatorname{SoC}\left(t_{f}\right)=0.65, m_{f}(0)=0, \\
& F_{b}(0)=F_{b}\left(t_{f}\right)=F_{g}(0)=F_{g}\left(t_{f}\right)=F_{h}(0)=F_{h}\left(t_{f}\right)=0 .
\end{aligned}
$$

Note that the charge sustainability is addressed in the present work by employing SoC boundary conditions, rather than introducing an additional term in the optimization cost function. This condition enables to make a fair comparison of fuel consumption between the solutions of different optimization methods, without having to refer to "equivalent fuel" quantification caused by unequal boundary $\mathrm{SoC}$ values between the different solutions, which may be inaccurate. Finally, $T_{b}(0)=T_{a m b}=25^{\circ} \mathrm{C}$ and $\mathrm{SoH}(0)=1$ are introduced.

c) Combined optimization of both vehicle speed and power split (OCP-Joint): In this framework, the OCP formulation includes the optimization of the power split (as in OCP-EM), however, the driving speed is unspecified, which leads to a formulation by which both EM and forward speed are simultaneously optimized. By this new formulation, the optimization problem becomes more complex and powerful than any of the individual optimization problems and it represents new knowledge. The state space model in this case is already specified in (11) with $\mathbf{x} \triangleq\left[m_{f}, \mathrm{SoC}, v, s, F_{g}, F_{b}, F_{h}\right]^{T}$, where $v$ is now included as a state according to (2), and (3) does not represent an algebraic constraint as in OCP-EM. The objective of the OCP-Joint is to minimize $J=m_{f}\left(t_{f}\right)$ analogously to the OCP-EM by finding three inputs $\mathbf{u}=\left[j_{b}, j_{g}, j_{h}\right]^{T}$, however the speed profile $v$ now also results from the optimization, as a consequence of the jerk inputs $\mathbf{u}$. The inequality constraints and the boundary conditions in this case collect all the conditions (except (18)) imposed in OCP-S and OCP-EM.

d) Two-step optimization of vehicle speed and power split (OCP-2-step): The (OCP-2-step) simply obtains the speed profile by solving OCP-S and combining that a posteriori with OCP-EM. OCP-2-step will be used in Section IV to benchmark OCP-Joint (for $\rho=0$, OCP-2-step is precisely related to existing optimization schemes in the literature).

The foregoing OCPs may be solved by various methods in different categories, such as dynamic programming, direct methods and indirect methods. In this work, these OCPs are solved by an indirect method embedded OCP solver (named PINS) that is based on C++ [28]. This method solves the optimization problem by converting it into a boundary value problem resulting from Pontryagin's minimum principle (PMP). Path constraints (for example, battery current limits, acceleration diamond and energy source power limits) are not explicitly included, instead, the inequalities are approximated and included in the Lagrange term $\int_{0}^{t_{f}} \mathcal{L}(\mathbf{x}, \mathbf{u}, t) d t$ by using smooth barrier functions. Non-smooth functions, such as $\operatorname{sign}(\cdot)$ are smoothed by approximations. The smoothness of the various approximation functions is freely tunable, subject to a well-known trade-off between accuracy and robustness (convergence to a solution) of the solver. It has been shown in [29] that this indirect solver offers comparable performance to solvers pertaining to other categories in terms of accuracy, robustness and computational speed.

\section{Simulation Results}

In this Section the OCPs defined in Section III are solved for the specific vehicle mission shown in Fig. 7 that corresponds to a rural route with scarce traffic. This route has been deliberately chosen to minimize the traffic influence, which is not taken into account in this paper. Road geometry including

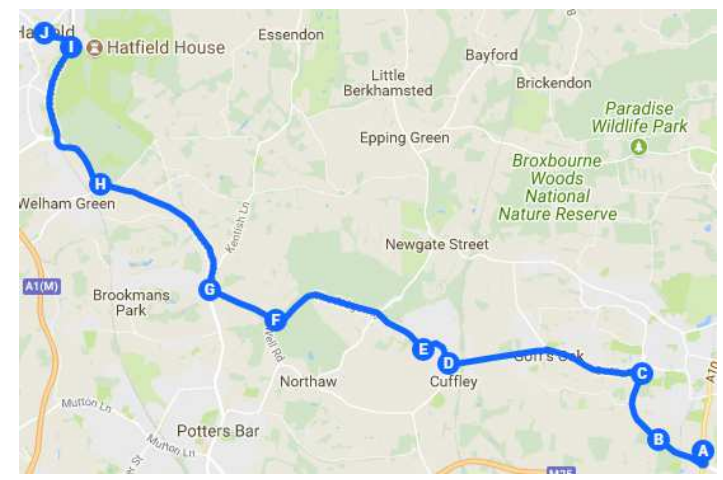

Fig. 7. $18.9 \mathrm{~km}$ rural route selected for the vehicle mission https://goo.gl/ytrVUM. 


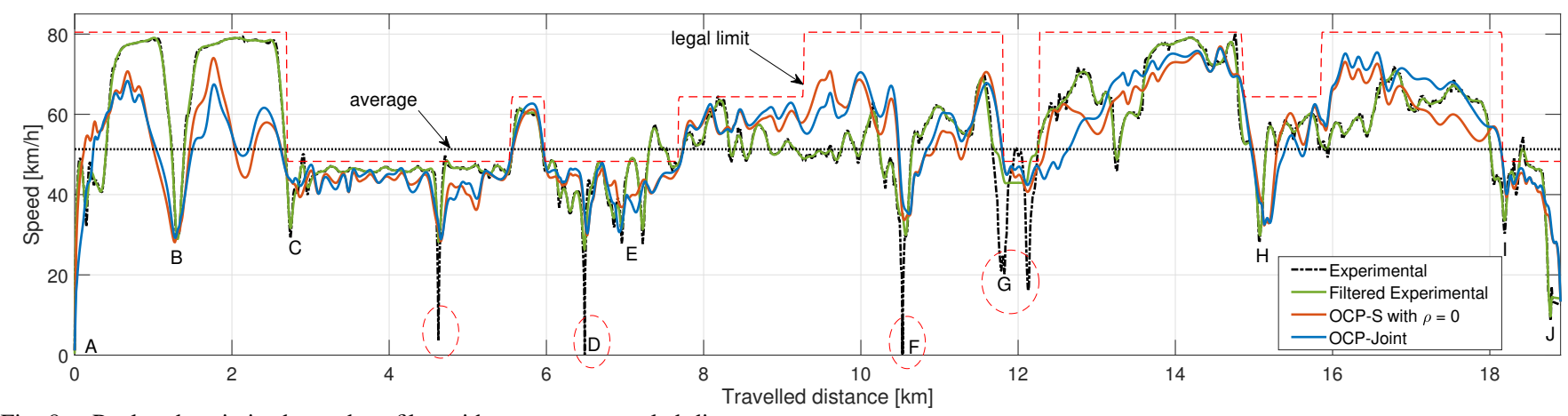

Fig. 8. Real and optimized speed profiles with respect to traveled distance.

the actual gradient is exported from Google MyMaps and it is then converted into the curvature model. The variable speed limit imposed throughout the journey captures the real conditions of the chosen path. Since traffic is not considered, the speed is not constrained to the behavior of other vehicles.

\section{A. Driving speed optimization assessment against real driving}

With the aim of showing the importance of driving speed optimization in terms of fuel saving and investigating the associated impact on the battery temperature and $\mathrm{SoH}$, an unoptimized human-driver speed profile of a petrol passenger car for the route in Fig. 7 is utilized for comparison with the optimal solutions. OCP-S is deployed with $\rho=0$ to simulate a conventional vehicle, while the main idea OCP-Joint is also used for benchmarking. The comparative results are shown in Figure 8 , where the trip is requested to be accomplished in 1326 s (identical to the real case), which corresponds to the vehicle being driven at an average speed of $51.3 \mathrm{~km} / \mathrm{h}$ with identical initial and terminal speeds to the real case. Note that the sudden dips (highlighted by dashed ellipses) in the real profile are caused by isolated traffic, therefore for fair comparisons they are not taken into account; the 'modified real' speed profile, also shown in Fig. 8, is used instead, which is obtained by passing the real profile though a median filter to remove such very sharp changes in speed.

Compared to the real driving speed, the optimal profiles lead to more efficient vehicle operation (as will be quantified in Table II) by using the information of the upcoming road slopes and speed limit. For example, at the beginning of the journey, the human driver accelerates to the speed limit, and upon reaching it, decelerates dramatically because of the approaching junction at $1 \mathrm{~km}$. Conversely, the optimal profiles stop increasing at a much lower speed, and they are then followed by a milder deceleration to pass through the first corner. It is also noticed that at $7 \mathrm{~km}$, the human driver exceeds the legal speed limit by view of the sign ahead indicating the higher limit, whereas the optimized results do not suffer from such a human 'error' and strictly obey the legal speed limit at all times.

To further understand the nature of optimal as compared to real driving, two different cases of OCP-EM are solved by injecting: a) the optimal speed from OCP-S (OCP-2-step), and b) the modified real speed profile; and comparing these with the solutions of OCP-Joint in terms of fuel economy as well as battery temperature and SoH. In the latter OCP-EM case it may be argued that the real driving speed is collected from a conventional vehicle, which is not consistent with the hybrid powertrain architecture deployed in OCP-EM. However, the human driver may not significantly change his/her driving style when the conventional car is replaced by a hybrid one with similar engine power, as the driving style of a particular driver is less influenced by the powertrain architecture but highly depends on the maximum power that can be delivered.

The results in terms of battery lifetime, and average fuel consumption are shown in Table II, while Fig. 9 shows the battery current and temperature profiles for the three optimization cases. The available distance the vehicle can travel before

TABLE II

COMPARISON AMONG DIFFERENT OPTIMIZATION APPROACHES

\begin{tabular}{lrr}
\hline \hline & $\begin{array}{r}\text { Battery life } \\
{[\mathrm{km}]}\end{array}$ & $\begin{array}{r}\text { Fuel economy } \\
{[\mathrm{L} / 100 \mathrm{~km}]}\end{array}$ \\
\hline OCP-Joint & 157500 & 3.84 \\
OCP-2-step & 127700 & 4.08 \\
Exp. speed+OCP-EM & 89573 & 5.92 \\
\hline \hline
\end{tabular}
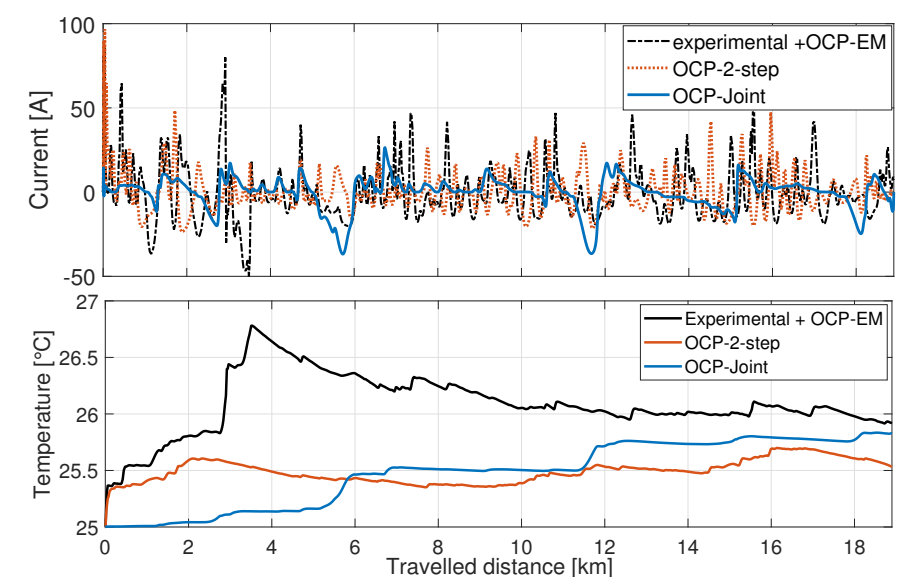

Fig. 9. Battery current and temperature profiles with $T_{a m b}=25^{\circ} \mathrm{C}$.

the battery reaches end-of-life is calculated according to the degradation suggested by the end of the present mission. It is shown that the OCP-Joint beyond substantially improved fuel economy, offers enhanced battery durability compared to the other two cases, as OCP-Joint is the most bounded throughout the simulation in terms of positive and negative 
current maximum values. The beneficial behavior of OCPJoint in terms of battery current and consequently in terms of $\mathrm{SoH}$, together with the relevance of temperature variations, is explained as follows.

From the battery thermal model (9), $T_{b}$ is affected by $i_{b}$ and the convective cooling (subject to the temperature difference from ambient temperature). As such, it may be the case that up to a certain amount of battery current there is no visible change in battery temperature because the resistive heat $R_{b} i_{b}^{2}$ is suppressed by the cooling effect, while only even higher operating currents will increase $T_{b}$. As illustrated in Fig. 9, a large current spike always results in a significant step change in temperature. For example, the main temperature increase of OCP-2-step occurs at the very beginning when a large discharging current is requested. Comparing to the other cases, the OCP-Joint solution has overall smaller $i_{b}$, but the duration of its larger charging currents appearing at $5.5 \mathrm{~km}$ and $11.5 \mathrm{~km}$ is much longer. Hence, the terminal temperature in all optimization cases ends up being very similar.

Battery current on the other hand represents the only variable that influences $\mathrm{SoH}$, which is particularly proportional to the integration of $\left|i_{b}\right|$ (see (8)). Therefore, it is clear that a significant positive jump in the temperature value (for example the one appears at $2.5 \mathrm{~km}$ ) is certainly an indication of a large $i_{b}$ that will also lead to a dramatic SoH drop. Since all the three optimization cases are charge sustained, the integration of their current profiles over $\left[0, t_{f}\right]$ are all zero. This means that any large positive variations in the current (even in the form of spikes) will have to be compensated by negative variations, thus overall increasing the average value of $\left|i_{b}\right|$ and hence the SoH drop. A real driver tends to drive the vehicle with higher values of acceleration and deceleration than the optimal ones. Hence more power will be requested from the powewtrain, and most likely the battery itself, implying large current spikes, in the case of the experimental + OCP-EM case. Also, when the powertrain characteristics are not taken into account in the optimization, as in the case of OCP-2step, the speed profile solution tends to have the PnG form, in which the initial part of each PnG segment is large acceleration and therefore large battery current. In contrast, in OCP-Joint the optimization takes into account that large battery power amounts are less efficient than low power values, therefore it restricts the battery usage to lower current values.

\section{B. Assessment of driving speed combined optimization}

To gain more insight into the behavior of different optimization schemes and to expose the benefits of combined optimization, the next example is carried out to explicitly compare the simultaneously optimized results of OCP-Joint and the solutions of OCP-2-step. The same vehicle mission as defined in the last example is used with the same average speed and boundary speed values.

As it can be noticed in Fig. 10, the consideration of road elevation in the optimization mainly gives rise to an additive perturbation to the optimal speed solution in comparison to the flat road. In order to focus on and compare the main mechanisms that produce the nature of the optimized speed profiles, the elevation of the route is omitted for the subsequent analysis in this section. In Fig. 10, the speed profile solved by the joint optimization is compared with the solutions from the speed optimization by OCP-S for different vehicle topologies. The results show that the optimized speed profile is dependent upon the selected optimization strategy and also varies as $\rho$ changes when OCP-S is deployed. It can be seen that the optimal driving speed for the case $\rho=0$ (conventional vehicle with no braking energy recovery) follows a pattern named pulse-and-glide $(\mathrm{PnG})$, which consists of rapid acceleration until a maximum velocity is reached, followed by a period of coasting to pass through the next corner. Such a speed pattern has been proven to be the most fuel efficient strategy in conventional vehicles [11]. This phenomenon of PnG fades away in the optimal solutions as the powertrain shifts towards the electric architecture that allows more regenerative brakes by increasing $\rho$. Eventually, it is hardly observed in the optimal profile when $\rho=1$ (hybrid vehicle with braking energy recovery), because the power amounts requested for acceleration and deceleration are balanced to take advantage of the fully recoverable braking energy. Moreover, the speed solution produced by the conventional powertrain formulation is closer to the simultaneously optimized speed (OCP-Joint) than that of the electric powertrain case in terms of root mean square (RMS) difference. The reason is that in the proposed architecture the battery is only a minor energy source, giving rise to a hybrid powertrain closer to the conventional type.

To complete the comparison, the speed profiles determined by the OCP-S speed optimization respectively for $\rho=0,0.2,0.4,0.6,0.8$, and 1 are fed to OCP-EM (leading to five cases of OCP-2-step), the solutions of which including the power split and the corresponding average fuel consumption are compared with their counterpart found by the combined optimization scheme OCP-Joint. The comparative results are reported in Fig. 11. It can be seen that the proposed OCP-Joint strategy benefits the fuel economy by at least $1.3 \%$ due to the inclusion of the actual hybrid powertrain in the optimization. Although the hybrid powertrain is neglected in OCP-S, the optimality of the solution in that case can be enhanced by manipulating $\rho$ to allow a suitable amount of regenerative braking. In the present case, $\rho=0.4$ gives the closest solution to OCP-Joint in terms of fuel consumption.

Apart from the fuel economy, the simultaneous optimization also improves the battery thermal behavior and durability, which can be inferred from the battery operating current, analogously to the previous example in Section IV-A. In Fig. 12, the current profile of the OCP-Joint is compared with two cases pertaining to the OCP-2-step respectively with $\rho=0$ and $\rho=1$. Due to the removal of the elevations, the relationship between battery current, and consequently temperature and $\mathrm{SoH}$ can be more clearly observed in this case. It is clear that the OCP-Joint produces the most bounded $i_{b}$, with also the fewest fluctuations throughout the mission, thereby the solution of OCP-Joint tends to deliver enhanced battery thermal and SoH performance. It can be seen in Fig. 11 that the solution of OCP-Joint is able to restrain the increase of battery temperature by about $12 \%$ as compared to the most fuel efficient OCP-2-step case $(\rho=0.4)$, and the benefit is 


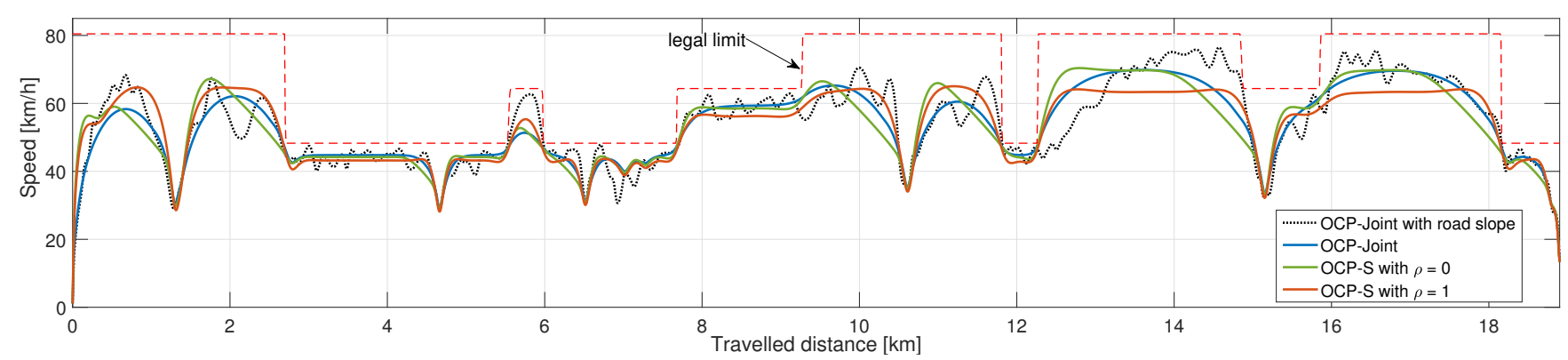

Fig. 10. Optimal speed profile under different scenarios. The values of RMS difference between the profiles obtained by OCP-Joint and OCP-S respectively with $\rho=0$ and $\rho=1$ are 3.83 and 4.14 when road elevation is ignored.

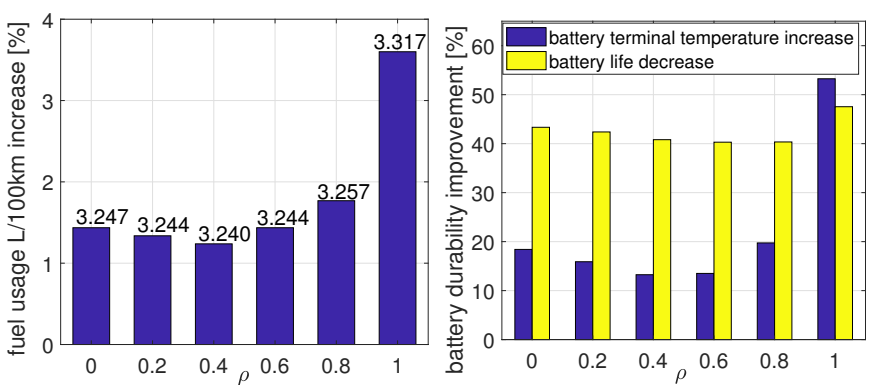

Fig. 11. Benefit of OCP-Joint compared to OCP-2-step with different $\rho$ in terms of average fuel consumption (fuel economy is shown on top of each bar in terms of $\mathrm{L} / 100 \mathrm{~km}$ ) and battery durability when road elevation is ignored.

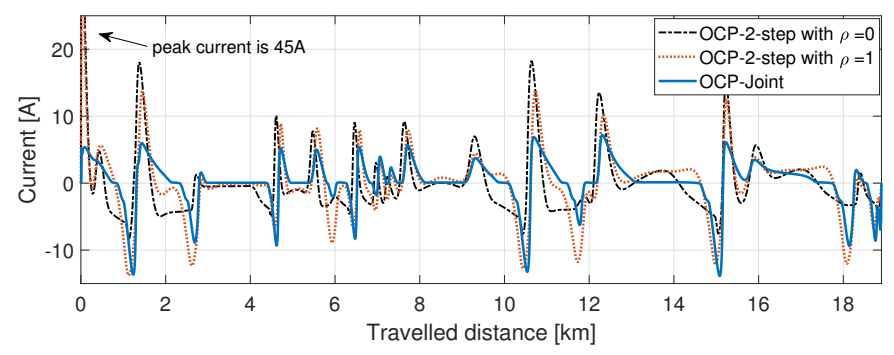

Fig. 12. Battery current profiles when road elevation is ignored.

increased when $\rho$ diverges from 0.4. The depleted $\mathrm{SoH}$ is almost steady for different choices of $\rho$, which amounts to the battery life being saved by approximately $40 \%$ by the combined optimization.

Finally, the best behaved OCP-2-step case with $\rho=0.4$ is selected for further comparison with the simultaneously optimized solution of OCP-Joint. In this case the RMS difference between the two optimized speed profiles is further reduced to 3.46 , which is lower than the differences calculated for other values of $\rho$. In Fig. 13, the battery SoC and power split obtained in both scenarios are compared. The main difference between the two optimal solutions is that the individually optimized speed has a higher acceleration after passing a corner, therefore in the acceleration phase it requests higher propulsive power, which is mainly provided by the ICE, while the battery provides an additional, small amount of power. By observing the SoC, the battery is depleted more for the OCP2-step at the beginning of the mission to fulfill the power demand requested by the associated speed profile. To remain strictly charge sustaining, less battery is used for the OCP-2step case at the end of the mission while both scenarios have similar behavior in between.
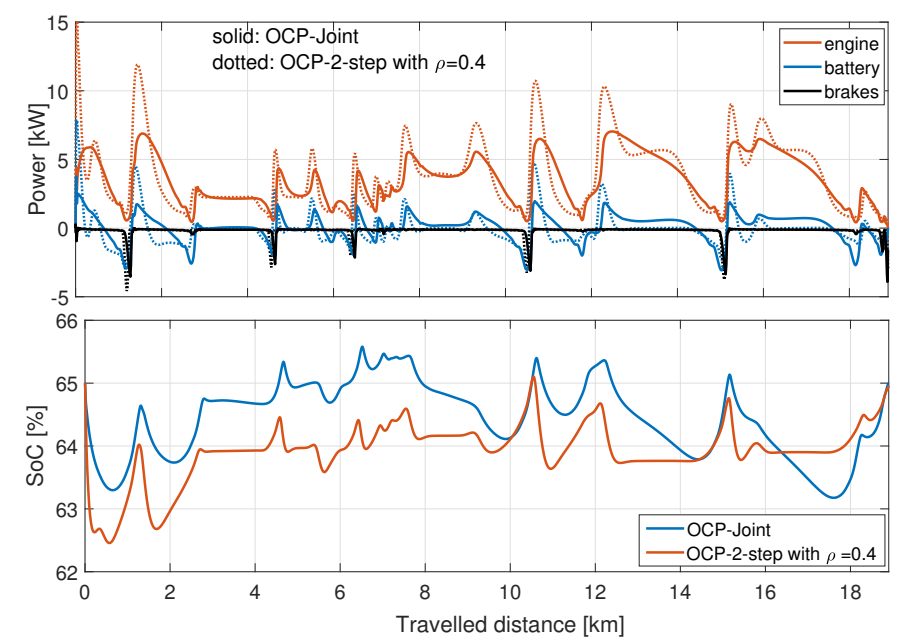

Fig. 13. Comparison of OCP-Joint and OCP-2-step with $\rho=0.4$ in terms of (Top) power flow of engine $P_{g}$, battery $P_{b}$, mechanical brakes $P_{h}$ and (Bottom) battery SoC when road elevation is ignored.

\section{CONCLUSIONS}

The energy management control and the driving speed optimization of a series HEV in the context of fuel economy are addressed in this paper by an optimal control methodology. The novelty of the proposed method is that it solves the optimal speed profile and the power split simultaneously, with the drive mission including only the path, the specified traveling time and constraints that address safety and comfort. The less complex sole optimization scheme of the vehicle speed and the energy management respectively are also presented and combined, forming a two-step scheme with the same overall objective as the proposed simultaneous optimization scheme, to benchmark against the proposed strategy in terms of fuel efficiency and battery durability. It is shown that the simultaneous optimization is solvable and it performs better in terms of fuel economy while also improving battery lifetime. Nevertheless, the optimality of the two-step methodology can be improved by suitably tuning the objective function for the speed optimization via a parameter that is associated with the regenerative braking capability of the powertrain.

From a technological perspective, the presented methodology works in real time as implemented in the PINS suite and requires as input only basic information on the vehicle state and route characteristics that are easily available on a navigation system. It can also be used for benchmarking purposes for a system where the computation power is rigorously limited. 
Future research efforts will be devoted to the extension of the methodology to the case where traffic conditions and other mission uncertainties are taken into consideration.

\section{REFERENCES}

[1] Y. Wang and Z. Sun, "Dynamic analysis and multivariable transient control of the power-split hybrid powertrain," IEEE/ASME Transactions on Mechatronics, vol. 20, no. 6, pp. 3085-3097, 2015.

[2] C. M. Martinez, X. Hu, D. Cao, E. Velenis, B. Gao, and M. Wellers, "Energy management in plug-in hybrid electric vehicles: Recent progress and a connected vehicles perspective," IEEE Transactions on Vehicular Technology, vol. 66, no. 6, pp. 4534-4549, 2017.

[3] J. M. Lujan, C. Guardiola, B. Pla, and A. Reig, "Analytical optimal solution to the energy management problem in series hybrid electric vehicles," IEEE Transactions on Vehicular Technology, vol. 67, no. 8, pp. 6803-6813, 2018.

[4] T. Liu, X. Hu, S. E. Li, and D. Cao, "Reinforcement learning optimized look-ahead energy management of a parallel hybrid electric vehicle," IEEE/ASME Transactions on Mechatronics, vol. 22, pp. 1497-1507, 2017.

[5] N. Kim, S. Cha, and H. Peng, "Optimal control of hybrid electric vehicles based on pontryagin's minimum principle," IEEE Transactions on Control Systems Technology, vol. 19, no. 5, pp. 1279-1287, 2011.

[6] L. Serrao, S. Onori, and G. Rizzoni, "ECMS as a realization of pontryagin's minimum principle for HEV control," in Proceedings of American Control Conference, 2009, pp. 3964 -3969.

[7] W. Shabbir and S. A. Evangelou, "Exclusive operation strategy for the supervisory control of series hybrid electric vehicles," IEEE Transactions on Control Systems Technology, vol. 24, no. 6, pp. 2190-2198, 2016.

[8] B. Chen and S. Evangelou, "Truncated battery power following strategy for energy management control of series hybrid electric vehicles," in European Control Conference, 2019.

[9] J. Liu, Y. Chen, J. Zhan, and F. Shang, "Heuristic dynamic programming based online energy management strategy for plug-in hybrid electric vehicles," IEEE Transactions on Vehicular Technology, vol. 68, no. 5, pp. 4479-4493, 2019.

[10] Y. L. Murphey, J. Park, L. Kiliaris, M. L. Kuang, M. A. Masrur, A. M. Phillips, and Q. Wang, "Intelligent hybrid vehicle power control part ii: Online intelligent energy management," IEEE Transactions on Vehicular Technology, vol. 62, no. 1, pp. 69-79, 2013.

[11] S. E. Li, H. Peng, K. Li, and J. Wang, "Minimum fuel control strategy in automated car-following scenarios," IEEE Transactions on Vehicular Technology, vol. 61, no. 3, pp. 998-1007, 2012.

[12] S. Uebel, N. Murgovski, C. Tempelhahn, and B. Baker, "A two-level $\mathrm{mpc}$ for energy management including velocity control of hybrid electric vehicles," IEEE Transactions on Vehicular Technology, vol. 68, no. 6, pp. 5494-5505, 2019.

[13] R. Lot and S. A. Evangelou, "Green driving optimization of a series hybrid electric vehicle," in Conference on Decision and Control, 2013.

[14] X. Yan, J. Fleming, C. Allison, and R. Lot, "Portable automobile data acquisition module (adam) for naturalistic driving study," in 15th EAEC European Automotive Congress, 2017.

[15] N. Mohan, T. M. Undeland, and W. P. Robbins, Power Electronics: Converters, Applications, and Design. USA: John Wiley \& Sons, 2002.

[16] S. A. Evangelou and A. Shukla, "Advances in the modelling and control of series hybrid electric vehicles," in Proceedings of American Control Conference, 2012.

[17] P. Pillay and R. Krishnan, "Modelling, simulation, and analysis of permanent-magnet motor drives, Part I: The permanent-magnet synchronous motor drive," IEEE Transactions on Industry Application, vol. 25, no. 2, pp. 265-273, 1989.

[18] L. Johannesson, N. Murgovski, S. Ebbesen, B. Egardt, E. Gelso, and J. Hellgren, "Including a battery state of health model in the hev component sizing and optimal control problem," in 7th IFAC Symposium on Advances in Automotive Control, 2013, pp. 398-403.

[19] D. J. Auger, M. F. Groff, G. Mohan, S. Longo, and F. Assadian, "Impact of battery ageing on an electric vehicle powertrain optimisation," Journal of Sustainable Development of Energy, Water and Environment System, vol. 2, no. 4, pp. 350-361, 2014.

[20] S. J. Moura, J. L. Stein, and H. K. Fathy, "Battery-health conscious power management in plug-in hybrid electric vehicles via electrochemical modeling and stochastic control," IEEE Transactions on Control Systems Technology, vol. 21, no. 3, pp. 679-694, 2013.
[21] S. Chacko and Y. M. Chung, "Thermal modelling of li-ion polymer battery for electric vehicle drive cycles," Journal of Power Sources, vol 213, pp. 296-303, 2012.

[22] "Electrically propelled road vehicles - Test specification for lithium-ion traction battery packs and systems - Part 1: High-power applications," ISO, Standard, 2011.

[23] Y. Kim, S. Mohan, J. B. Siegel, A. G. Stefanopoulou, and Y. Ding, "The estimation of temperature distribution in cylindrical battery cells under unknown cooling conditions," IEEE Transactions on Control Systems Technology, vol. 22, no. 6, pp. 2277-2286, 2014.

[24] H. Park, "A design of air flow configuration for cooling lithium ion battery in hybrid electric vehicles," Journal of Power Sources, vol. 239, pp. 30-36, 2013.

[25] M. Pahlevaninezhad, P. Das, J. Drobnik, P. K. Jain, and A. Bakhshai, "A novel zvzcs fullbridge dc/dc converter used for electric vehicles," IEEE Trans. on Power Electronics, vol. 27, no. 6, pp. 2752-2769, 2012.

[26] F. Biral, M. Da Lio, and E. Bertolazzi, "Combining safety margins and user preferences into a driving criterion for optimal control-based computation of reference maneuvers for an adas of the next generation," IEEE Intelligent Vehicles Symposium, pp. 36-41, 2005.

[27] B. Chen, S. Evangelou, and R. Lot, "Fuel efficiency optimization methodologies for series hybrid electric vehicles," in IEEE Vehicle Power and Propulsion Conference (VPPC), 2018.

[28] E. Bertolazzi, F. Biral, and M. D. Lio, "Real-time motion planning for multibody systems: Real life application examples," Multibody System Dynamics, vol. 17, no. 2-3, pp. 119-139, 2007.

[29] F. Biral, E. Bertolazzi, and P. Bosetti, "Notes on numerical methods for solving optimal control problems," IEEJ Journal of Industry Applications, vol. 5, no. 2, pp. 154-166, 2016.

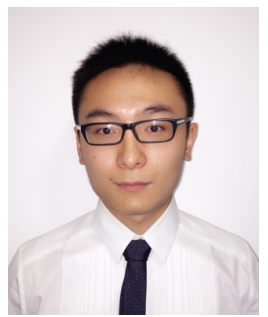

Boli Chen (M'16) received the B. Eng. in Electrical and Electronic Engineering in 2010 from Northumbria University, UK. In 2011 and 2015, he respectively received the MSc and the Ph.D. in Control Systems from Imperial College London, UK. Currently, he is a Lecturer in the Department of Electronic and Electrical Engineering, University College London, U.K. His research focuses on control, optimization, estimation and identification of a range of complex dynamical systems, mainly from automotive and power electronics areas.

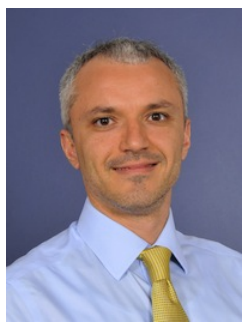

Simos A. Evangelou (M'05-SM'17) received the B.A./M.Eng. degree in electrical and information sciences from the University of Cambridge, Cambridge, U.K., and the Ph.D. degree in control engineering from Imperial College London, London, U.K.. He is currently a Reader with the Department of Electrical and Electronic Engineering, Imperial College London, London, U.K. Dr. Evangelou is a Member of IFAC Technical Committee Automotive Control and on the editorial board of international journals and conferences, including the IEEE Control Systems Society Conference Editorial Board.

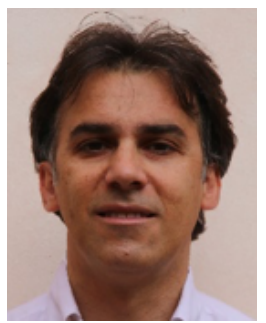

Roberto Lot is Professor of Automotive Engineering at the University of Southampton (UK) since 2014. He received a PhD in Mechanics of Machines in 1998 and a Master Degree cum laude in Mechanical Engineering in 1994 from the University of Padova (Italy). His research interests include dynamics and control of road and race vehicles, contributing to make our vehicles safer, faster, and eco-friendlier. He has directed several national and international research projects and published more than 100 scientific papers. 